Original paper

\title{
FIB-5 versus FIB-4 index for assessment of hepatic fibrosis in chronic hepatitis B affected patients
}

\author{
Khaled Metwally', Maha Elsabaawy', Mohamed Abdel-Samiee', Wessam Morad², Nermine Ehsan ${ }^{3}$, Eman Abdelsameea' \\ 'Hepatology and Gastroenterology Department, National Liver Institute, Menoufia University, Egypt \\ ${ }^{2}$ Community and Preventive Medicine Department, National Liver Institute, Menoufia University, Egypt \\ ${ }^{3}$ Pathology Department, National Liver Institute, Menoufia University, Egypt
}

\begin{abstract}
Aim of the study: Chronic hepatitis B virus (HBV) infection is one of the major health problems worldwide. Use of non-invasive tests for assessment of hepatic fibrosis such as the FIB-4 index could be used to avoid liver biopsy. Another promising noninvasive test, FB- 5 , could also be used to detect significant hepatic fibrosis. The aim of the study was to compare the use of FIB-5 and FIB-4 as noninvasive markers to assess chronic HBV-related hepatic fibrosis.

Material and methods: This study was done on 176 chronic HBV patients who underwent liver biopsy. Grading and staging of liver fibrosis was done according to the METAVIR scoring system. FIB-5 and FIB-4 scores were calculated for all patients.

Results: As regards FIB-4 for differentiation between non-significant fibrosis (group I) and significant fibrosis (group II), at a cutoff level of 1.28 with positive predictive value (PPV) $41.4 \%$ and specificity $48 \%$ while at a cutoff level of 7.08 with PPV $98.8 \%$ and specificity $98 \%$ for FIB-5.

Conclusions: As regards both scores, the FIB-5 score was more specific than FIB-4 for diagnosing significant from nonsignificant hepatic fibrosis in patients with chronic HBV infection.
\end{abstract}

Key words: FIB-5, FIB-4, fibrosis, biopsy, chronic HBV.

Address for correspondence:

Dr. Khaled Metwally, Assoc. Prof. of Hepatology and Gastroenterology Department, National Liver Institute, Menoufia University, Egypt, 32511, phone: 00201000486019 , fax: +20 48 2222740, e-mail: kh_m55555@yahoo.com

\section{Introduction}

Liver cirrhosis is a critical complication of chronic liver diseases. Early diagnosis is mandatory for management and surveillance of patients with chronic liver disease. Diagnosis of liver cirrhosis was based mainly on liver biopsy, which is an invasive procedure with rare but potentially life-threatening complications and also prone to sampling errors. Use of non-invasive laboratory and radiological methods has rapidly decreased the use of liver biopsy for diagnosing liver cirrhosis in patients with chronic viral hepatitis. These methods are widely used in many clinical practices and recommended by the European Association for the Study of the Liver (EASL) and international guidelines [1-5]. Non-invasive markers such as FIB-4 use age, alanine aminotransferase (ALT), aspartate aminotransferase (AST) and platelet count. Recently FIB-5 using albumin, alkaline phosphatase (ALP), AST to ALT ratio and platelet count has been used for assessment of liver fibrosis and for predicting severe fibrosis or cirrhosis in patients with chronic hepatitis $\mathrm{C}$ infection [6, 7]. FIB-5 and FIB-4 use for assessment of nonsignificant (F0-1) and significant fibrosis (F2-4) in patients with chronic hepatitis $\mathrm{B}$ virus (HBV) was the aim of this study.

\section{Material and methods}

This cross-sectional study was conducted on $176 \mathrm{pa}-$ tients with chronic HBV infection who were recruited from outpatient clinics of the National Liver Institute Hospital, Menoufia University from June 2016 to May 
2018. Written informed consent from each patient in the study especially for liver biopsy, with approval of the local ethical committee, was obtained before starting the data collection. The study protocol conformed to the ethical guidelines of the 1975 Declaration of Helsinki as reflected in a priority approval by the institution's human research committee. Exclusion criteria of the study included patients coinfected by hepatitis $\mathrm{C}$ virus (HCV), hepatitis D virus (HDV) or human immunodeficiency virus (HIV), patients with primary or secondary liver tumors, patients who received any

Table 1. Frequency distribution of all studied patients according to METAVIR scoring system

\begin{tabular}{lcc}
\hline Stage & Frequency $(\boldsymbol{n})$ & Percent $(\%)$ \\
\hline F0 & 1 & 0.6 \\
\hline F1 & 97 & 55.1 \\
\hline F2 & 61 & 34.7 \\
\hline F3 & 9 & 5.1 \\
\hline F4 & 8 & 4.5 \\
\hline
\end{tabular}

Table 2. Baseline characteristics of chronic hepatitis B patients with hepatic fibrosis regards two main classifications $(N=176)$

\begin{tabular}{|c|c|c|c|}
\hline Parameters & $\begin{array}{c}\text { Non-significant } \\
\text { fibrosis (F0-1) } \\
(n=98)\end{array}$ & $\begin{array}{c}\text { Significant fibrosis } \\
(F 2-4) \\
(n=78)\end{array}$ & $P$-value \\
\hline Age (years) & $32.37(9.23)$ & $36.28(10.25)$ & $0.008^{*}$ \\
\hline $\begin{array}{l}\text { Total bilirubin } \\
\text { (mg/dl) }\end{array}$ & $0.76(0.25)$ & $0.81(0.44)$ & 0.6 \\
\hline ALT (U/I) & $35.73(20.07)$ & $43.79(30.15)$ & 0.15 \\
\hline AST (U/I) & $33.46(19.83)$ & 39.05 (23.33) & 0.12 \\
\hline $\operatorname{ALP}(U / I)$ & $68.87(15.68)$ & $96.73(30.86)$ & $0.00004^{*}$ \\
\hline GGT (mg/dl) & $29.65(10.63)$ & $42.38(21.08)$ & 0.00001 * \\
\hline Albumin (gm/dl) & $4.57(0.43)$ & $4.26(0.47)$ & $0.00002^{*}$ \\
\hline $\mathrm{HB}(\mathrm{g} / \mathrm{dl})$ & $14.9(10.4)$ & $13.45(1.51)$ & $0.04^{*}$ \\
\hline WBCs $\left(\times 10^{3} / \mathrm{mm}^{3}\right)$ & $6.49(1.65)$ & $6.34(1.72)$ & 0.54 \\
\hline $\begin{array}{l}\text { Platelets } \\
\left(\times 10^{3} / \mathrm{mm}^{3}\right)\end{array}$ & $222.66(51.26)$ & 193.54 (59.09) & $0.001^{*}$ \\
\hline$\gamma$-globulin (g/dl) & $1.3(0.2)$ & $1.53(0.43)$ & 0.00001 * \\
\hline PC (\%) & $91.86(6.74)$ & $87.08(7.94)$ & $0.00003 *$ \\
\hline INR & $1.08(0.07)$ & $1.13(0.08)$ & $0.00002^{*}$ \\
\hline $\begin{array}{l}\text { HBV DNA level } \\
(\mathrm{U} / \mathrm{l})\end{array}$ & $\begin{array}{c}12620011.88 \\
(95725298.66) \\
\end{array}$ & $\begin{array}{c}622951.35 \\
(2843934.18) \\
\end{array}$ & 0.48 \\
\hline AST/ALT ratio & $1.11(0.34)$ & $1.12(0.36)$ & 0.89 \\
\hline FIB-4 & $1.5(0.73)$ & $2.11(1.49)$ & $0.001^{*}$ \\
\hline FIB-5 & $5.83(1.87)$ & $4.15(0.94)$ & $0.00001^{*}$ \\
\hline
\end{tabular}

ALT - alanine aminotransferase, AST - aspartate aminotransferase, ALP - alkaline phosphatase, GGT - $\gamma$-glutamyl transferase, $H B$ - hemoglobin, WBCs - white blood cells, $P C$ - prothrombin concentration, INR - international normalized ratio previous antiviral or immunosuppressive medications, and also patients who refused liver biopsy or having any contraindication to undergo liver biopsy and patients with decompensated cirrhosis. All patients in the study were subjected to full history taking, complete physical examination, and laboratory investigations which included: complete blood count, liver function tests and abdominal ultrasound. The FIB-5 score was calculated according to Attallah et al. [7] as follows: albu$\min (\mathrm{g} / \mathrm{l}) \times 0.3+$ platelet count $\left(10^{9} / \mathrm{l}\right) \times 0.05-$ alkaline phosphatase $(\mathrm{IU} / \mathrm{l}) \times 0.014+\mathrm{AST} / \mathrm{ALT}$ ratio $\times 6+14$. The FIB-4 [8] score was calculated as follows: [Age $($ year $) \times \operatorname{AST}(\mathrm{IU} / \mathrm{l})] /\left[\right.$ platelet count $\left(\times 10^{9} / \mathrm{l}\right) \times \mathrm{ALT}$ $\left.(\mathrm{IU} / \mathrm{l})^{1 / 2}\right]$. All studied patients were positive for HBsAg for more than six months with a detectable HBV DNA level (IU/l) by real-time polymerase chain reaction (PCR) for diagnosis of chronic HBV infection. Liver biopsy sections were examined histopathologically blindly by an expert pathologist. Stages of fibrosis (F0-4) were assessed according to the METAVIR scoring system [9]. We further divided fibrosis stages into two groups: group I as F0-1 (non-significant fibrosis), and group II as F2-4 (significant fibrosis) [9].

\section{Statistical analysis}

Demographic data, clinical, laboratory and virologic data were collected. All patient data were tabulated and processed using SPSS version 22. Pearson correlation was conducted to correlate continuous parameters. Multivariate backward stepwise binary logistic regression analysis with significant fibrosis (F2) - as the dependent factor - was performed to determine how well the FIB- 5 test compared to FIB- 4 as a diagnostic test which can predict that a patient has nonsignificant (F0-1) or severe fibrosis (F3-4). Efficiency is an overall estimate of a test's ability to classify patients correctly. It is estimated by adding the number of the two correct classifications (true positive and true negative) and dividing by the total number of patients assessed. $P$-values $<0.05$ were considered significant.

\section{Results}

As regards liver fibrosis stage by histopathological examination, F0 was found in $1(0.6 \%), \mathrm{F} 1$ in 97 $(55.1 \%), \mathrm{F} 2$ in $61(34.7 \%), \mathrm{F} 3$ in $9(5.1 \%)$ and F4 in $8(4.5 \%)$ patients (Table 1$)$. There was a statistically significant difference between the two studied groups regarding age $(p=0.008)$. Non-significant fibrosis was diagnosed mostly in younger patients (Table 2). Serum albumin, platelet count, ALP, $\gamma$-glutamyl transferase (GGT), prothrombin concentration (PC) and interna- 


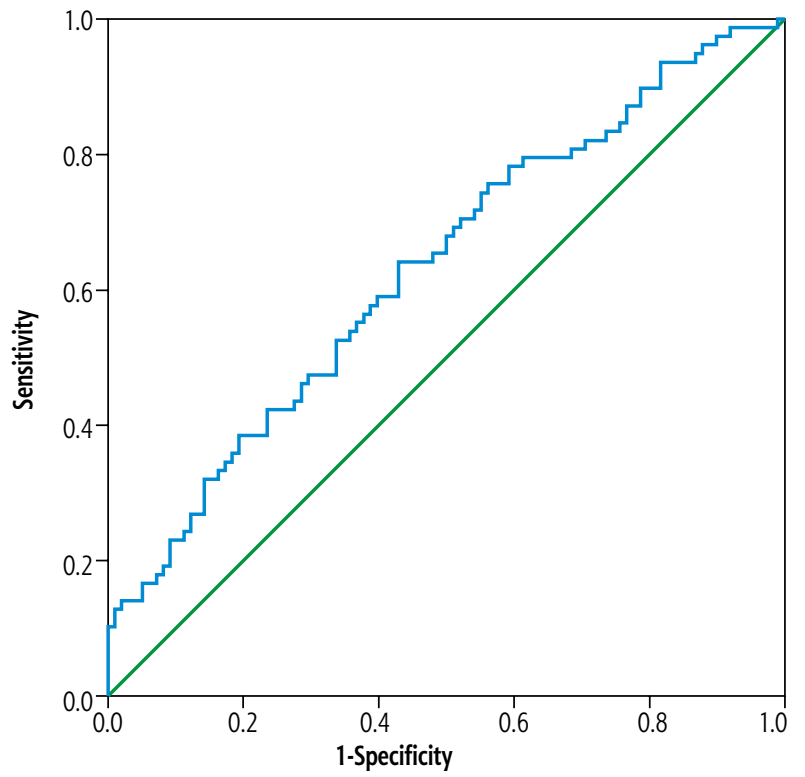

Fig. 1. Receiver-operating characteristic (ROC) curve generated by FIB-4 for differentiation between significant and non-significant fibrosis

tional normalized ratio (INR) were significantly different between groups (Table 2). ALP, albumin, and platelet count decreased in the group of advanced fibrosis. FIB-5 value and FIB-4 significantly differentiated between fibrosis groups ( $p=0.00001,0.001$ respectively) as shown in Table 2. There was a significant relationship between fibrosis stages and both serum indices. There was a significant increase in the level of FIB-4 as fibrosis progressed from non-significant (F0-1) to significant fibrosis (F2-4). A significant decrease in the level of FIB-5 ( $p=0.00001$ ) was observed with the progression of fibrosis stages from non-significant to significant fibrosis (Table 2). The area under the curve (AUC) values ( $p$-values) of the serum non-invasive indices are shown in Figures 1 and 2. The AUC of FIB-5 for differentiating non-significant fibrosis from significant fibrosis (as shown in Fig. 2) was $0.8(p<0.01)$ and for FIB-4, as shown in Figure 1, it was 0.63 $(p<0.01)$. When compared to liver biopsy, FIB-5 values at a cutoff level 7.08 showed a positive predictive value (PPV) of $98.8 \%$ to differentiate between F0, F1 and other fibrosis stages with specificity of $98 \%$ and FIB-4 values at a cutoff level of 1.28 showed a PPV of $41.4 \%$ to differentiate between F0, F1 and other fibrosis stages with specificity of $48 \%$ (Table 3 ).

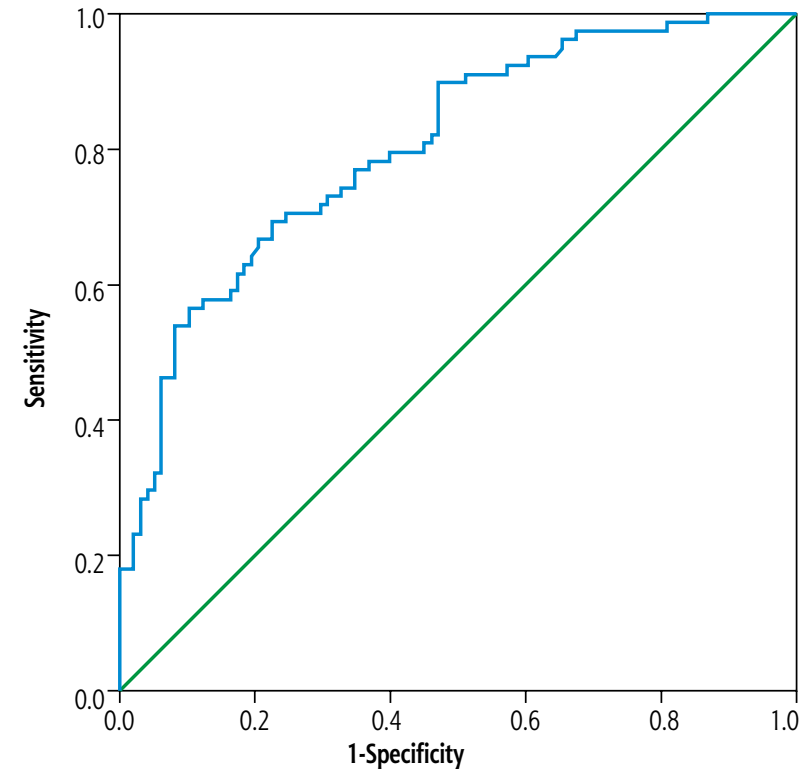

Fig. 2. Receiver-operating characteristic (ROC) curve generated by FIB-5 for differentiation between significant and non-significant fibrosis

\section{Discussion}

Liver biopsy is considered the traditional reference standard for fibrosis staging [10]. FIB-5 is a promising noninvasive test used for assessment of hepatic fibrosis [11]. The aim of this study was to compare the use of FIB-5 vs. FIB-4 for the differentiation between significant (F2-4) and non-significant hepatic fibrosis (F0-1) which was conducted on 176 patients with chronic hepatitis $B$ infection. A significant decrease in the level of FIB-5 ( $p=0.00001)$ was observed with the progression of fibrosis stages from non-significant (98 patients) to significant fibrosis (78 patients). FIB-5 values at a cutoff level of 7.08 showed a PPV of $98.8 \%$ with a specificity of $98 \%$ for the differentiation between significant and non-significant fibrosis. This high specificity and PPV compared to the FIB-4 value at a cutoff level of 1.28 with PPV of $41.4 \%$ and specificity of $48 \%$ are needed for taking treatment decisions in clinical practice. In this study, the AUROC of FIB- 4 to differentiate significant $(F 2-4, n=78)$ from non-significant fibrosis (F0-1, $n=98$ ) was 0.63 , with a sensitivity of $70.5 \%$. The negative predictive value (NPV) was $70 \%$ and the PPV was $41.4 \%$. Better results

Table 3. Performance characteristics of both fibro fast (FIB-5) and FIB-4 to differentiate between F0, F1 and other fibrosis stages ( $N=176$ cases)

\begin{tabular}{lccccccc}
\hline & AUC & Sensitivity (\%) & Specificity (\%) & PPV (\%) & NPV (\%) & SE & $95 \%$ Cl \\
\hline FIB-4 (1.28) & 0.63 & 70.5 & 48.0 & 41.4 & 70.0 & 0.04 & $0.55-0.72$ \\
\hline FIB-5 (7.08) & 0.80 & 23.1 & 98.0 & 98.8 & 46.2 & 0.03 & $0.74-0.87$ \\
\hline
\end{tabular}

AUC - area under the curve, PPV - positive predictive value, NPV - negative predictive value, SE - standard error 
were reported by Li et al. [12]. They obtained multiple meta-analysis evaluated the performance of FIB-4 in HBV mono-infected patients with 12 studies on 1908 patients and 10 studies on 2105 patients with the AUROCs ranging between 0.74 and 0.81 at a cutoff value between 1.45 and 1.62 and they concluded that the FIB-4 index is of great value for detecting significant fibrosis and also cirrhosis in HBV infected patients but its accuracy is suboptimal in exclusion of fibrosis and cirrhosis. Kim et al. [13] studied 575 patients with chronic HBV; they found that APRI and FIB-4 scores were not suitable to be used in clinical practice for assessment of hepatic fibrosis according to the Ishak staging system in chronic hepatitis B patients. Chayanupatkul et al. [14] concluded that the FIB-4 ROC curve value of 0.652 was suitable for diagnosing liver cirrhosis. Yin et al. [15] found that the best cutoff level of FIB-4 in identifying cirrhosis was $>2$. The FIB- 5 score in differentiation between significant and non-significant fibrosis in the present study differs from FIB 4 due to the presence of serum albumin, which was closely one of the synthetic functions of the liver, and also alkaline phosphatase, which represents hepatocellular integrity and excretory liver function $[11,13]$. FIB-5 is an easy, inexpensive, non-invasive, accurate and bedside test that could be used in clinical practice, and this needs further multiple studies on a large number of patients to confirm our results.

\section{Conclusions}

FIB-5 is a sensitive non-invasive test that might be used for diagnosis of significant and non-significant fibrosis in patients with chronic HBV infection. FIB-5 can help to decrease the use of liver of biopsies, which is an invasive procedure and needs to be avoided in clinical practice, and this is the main ultimate goal of the study. FIB-5, with a cutoff point of 7.08 , could be considered as a good and applicable marker. This will further need a large number of patients to be included in more studies to confirm it.

\section{Acknowledgements}

Many thanks for all staff members in Hepatology and Pathology Departments.

\section{Disclosure}

The authors declare no conflict of interest.

\section{References}

1. European Association for Study of Liver; Asociación Latinoamericana para el Estudio del Higado. EASL-ALEH Clinical Practice Guidelines: non-invasive tests for evaluation of liver disease severity and prognosis. J Hepatol 2015; 63: 237-264.

2. European Association for the Study of the Liver. EASL 2017 Clinical Practice Guidelines on the management of hepatitis B virus infection. J Hepatol 2017; 67: 370-398.

3. European Association for the Study of the Liver. EASL Recommendations on treatment of hepatitis C 2018. J Hepatol 2018; 69: 461-511.

4. European Association for the Study of the Liver (EASL), European Association for the Study of Diabetes (EASD), European Association for the Study of Obesity (EASO). EASL-EASDEASO Clinical Practice Guidelines for the management of the nonalcoholic fatty liver disease. J Hepatol 2016; 64: 1388-1402.

5. European Association for the Study of the Liver EASL clinical practical guidelines: management of alcoholic liver disease. J Hepatol 2012; 57: 399-420.

6. Degos F, Perez P, Roche B, et al. Diagnostic accuracy of FibroScan and comparison to liver fibrosis biomarkers in chronic viral hepatitis: a multicenter prospective study (the FIBROSTIC study). J Hepatol 2010; 53: 1013-1021.

7. Attallah AM, Shiha GE, Omran MM, et al. A discriminant score based on routine laboratory blood tests for accurate diagnosis of severe fibrosis and/or liver cirrhosis in Egyptian patients with chronic hepatitis C. Hepatol Res 2006; 34: 163-169.

8. Ahmad W, Ijaz B, Javed FT, et al. A comparison of four fibrosis indexes in chronic HCV: development of a new fibrosis-cirrhosis index (FCI). BMC Gastroenterol 2011; 11: 44 .

9. Ozyalvacli G, Kucukbayrak A, Kurt M, et al. Non-invasive fibrosis tests are correlated with necroinflammatory activity of liver in patients with chronic hepatitis B. Clin Ter 2014; 165 : e199-204.

10. Allam NA, El Sabawy MM, Abdelsamea E, et al. Noninvasive markers of liver disease in patients with $\mathrm{HBeAg}$ negative chronic hepatitis B and normal enzymes. Egyptian Liver J 2016; 5: 68-72.

11. Amorim TG, Staub GI, Lazzarotto C, et al. Validation and comparison of simple noninvasive models for the prediction of liver fibrosis in chronic hepatitis C. Ann Hepatol 2012; 11: 855-861.

12. Li Y, Chen Y, Zhao Y. The diagnostic value of FIB4 index for staging hepatitis B related fibrosis: A meta-analysis. PLoS One 2014; 9: e105728.

13. Kim WR, Berg T, Asselah T, et al. Evaluation of APRI and FIB4 scoring systems for non-invasive assessment of hepatic fibrosis in chronic hepatitis B patients. J Hepatol 2016; 64: 773-780.

14. Chayanupatkul M, Omino R, Mittal S, et al. Hepatocellular carcinoma in absence of cirrhosis in patients with chronic HBV infection. J Hepatol 2017; 66: 355-362.

15. Yin Z, Chen L, Zou J, et al. Diagnostic value of FIB 4 for liver fibrosis in patients with hepatitis B: a meta-analysis of the diagnostic test. Oncotarget 2017; 8: 22944-22953. 